\title{
Modelling of HTR Confinement Behaviour during Accidents Involving Breach of the Helium Pressure Boundary
}

\author{
Joan Fontanet, ${ }^{1}$ Luis E. Herranz, ${ }^{1}$ Alastair Ramlakan, ${ }^{2}$ and Lolan Naicker ${ }^{2}$ \\ ${ }^{1}$ Unit of Nuclear Safety Research, CIEMAT, Avenida Complutense, 2228040 Madrid, Spain \\ ${ }^{2}$ Pebble Bed Modular Reactor (Pty) Limited, 1279 Mike Crawford Avenue, 0046 Centurion, South Africa
}

Correspondence should be addressed to Joan Fontanet, joan.fontanet@ciemat.es

Received 30 January 2009; Revised 18 May 2009; Accepted 4 June 2009

Recommended by Jim Kuijper

Development of HTRs requires the performance of a thorough safety study, which includes accident analyses. Confinement building performance is a key element of the system since the behaviour of aerosol and attached fission products within the building is of an utmost relevance in terms of the potential source term to the environment. This paper explores the available simulation capabilities (ASTEC and CONTAIN codes) and illustrates the performance of a postulated HTR vented confinement under prototypical accident conditions by a scoping study based on two accident sequences characterized by Helium Pressure Boundary breaches, a small and a large break. The results obtained indicate that both codes predict very similar thermal-hydraulic responses of the confinement both in magnitude and timing. As for the aerosol behaviour, both codes predict that most of the inventory coming into the confinement is eventually depleted on the walls and only about $1 \%$ of the aerosol dust is released to the environment. The crosscomparison of codes states that largest differences are in the intercompartmental flows and the incompartment gas composition.

Copyright (C) 2009 Joan Fontanet et al. This is an open access article distributed under the Creative Commons Attribution License, which permits unrestricted use, distribution, and reproduction in any medium, provided the original work is properly cited.

\section{Introduction}

Modern High Temperature Reactors (HTRs) are designed in such a way that their inherent features provide adequate protection against hypothetical accidents. The main characteristics directly related to safety are the coated fuel particles (CFPs), the use of helium as a coolant, the passive decay heat removal, the negative temperature-reactivity coefficient, and a large margin between fuel operation and fuel damage temperature. As a result, HTRs do not require active safety systems or prompt operator actions to prevent any significant fuel failure or fission product release.

Helium has important plant safety implications from the point of view of plant confinement. Its noncondensable nature has two major implications: firstly, no large local temperature increases should be expected during anticipated operational occurrences (phenomena like departure from nucleate boiling cannot happen); secondly, the effectiveness of an LWR conventional containment gets substantially reduced since pressure suppression by condensation is ruled out. In the case of a primary circuit depressurization event, the helium would behave as a radionuclide carrier from the reactor cooling system, passing through the confinement building, to the environment.

Furthermore, the combination of high heat capacity, low power density, and high effective thermal conductivity in HTR cores results in slow thermal transients: fuel temperatures peak days after the initiating event. This means that in case of any delayed fission product release driven by high fuel temperatures, it would occur long after the depressurization was over. In the case of a confinement approach, at that time no helium would be available within the circuit to carry any fission product and aerosol from the primary system to the building. Therefore, a much lower offsite dose than in the case of an LWR would be expected. In the HTR context, filtration of the early release (i.e., dust and circulating activity in the HPB) becomes critical in reducing the source term to the environment.

Dust exiting the HPB will include fission and activation products so that the aerosol behaviour within the building is of utmost relevance in terms of the potential source term to the environment. Several phenomena, such as intercell flow, particle agglomeration, and settling, would be responsible for the radioactivity evolution within the 
confinement building. Hence, all those processes should be properly encapsulated in any analytical tool.

This paper illustrates, through a scoping study based on two postulated HPB break accidents, two key aspects of HTR safety analysis: predictability of current analytical tools and the performance of an alternate PBMR-type plant confinement. The predictability is discussed based on the range of validity of the models inside two accredited codes in the field of LWR safety analysis: ASTEC v1.3 [1] and CONTAIN 2.0 [2]. Both thermal-hydraulic and aerosol performance are reported according to estimates obtained by both codes. Furthermore, the code-to-code comparison highlights the strengths and weaknesses of these two codes.

\section{Models Applicability}

2.1. Particle Characterization. The applicability of the thermal-hydraulics and aerosol models encapsulated in ASTEC v1.3 and CONTAIN 2.0 for HTR safety analysis needs to be assessed.

The aerosol behaviour is closely linked to the dimensionless Knudsen number $\left(\mathrm{Kn}=2 \cdot \lambda / d_{p}\right)$, which conditions the medium resistance force to particle motion. According to its value, four different regimes are considered: continuum $(\mathrm{Kn} \ll 1)$, slip flow $(\mathrm{Kn}<0.25)$, transition $(0.1<\mathrm{Kn}<1.0)$, and free molecule $(\mathrm{Kn}>10)$ [3]. According to pressure and temperature ranges anticipated in HTR confinement accident scenarios (1-1.5 bar, 300-400 K, resp.) the mean free path would range between $10^{-7}-2.5 \cdot 10^{-7} \mathrm{~m}$ (pure helium) and $3 \cdot 10^{-8}$ to $9 \cdot 10^{-8} \mathrm{~m}$ (pure air). On the other side, AVR data [4] indicates that the expected particle size should be in the range of $10^{-6}$ to $10^{-5} \mathrm{~m}$. As a result, $\mathrm{Kn}$ may be readily estimated to be around 0.1 . In other words, particles would lay inside the limits of the slip flow regime even for pure helium. Only in the case of the smallest particles, Kn would increase up to 0.2 (helium-air mixture) or even 0.5 (pure helium), that is, right at the border slip flow and transition regimes.

Therefore, aerosol behaviour within the HTR confinement building during a postulated accident scenario should be described with models valid within the slip flow regime. Even in quite specific and extreme conditions, like those in the discharge compartment during a fast primary circuit depressurization, models capable of dealing with "slip-flow" conditions would still be suitable.

2.2. Aerosol Models. Most of the aerosol models included in ASTEC and CONTAIN are very similar since the models in both codes originate from the MAEROS code [5]. The key depletion and agglomeration models are presented next.

2.2.1. Depletion Models. Four deposition mechanisms are modelled into ASTEC and CONTAIN: gravitational settling, diffusion, thermophoresis, and diffusiophoresis.

(i) Gravitational deposition on the compartment floor is characterized by the settling velocity, $v_{\text {set }}$, which is given by the following expression [6]:

$$
v_{\mathrm{set}}=C_{C} \cdot \frac{\rho_{p} \cdot g \cdot d_{p}^{2}}{18 \cdot \mu_{g} \cdot \chi}
$$

This equation was derived based on the Stokes theory for small Reynolds numbers $\left(\operatorname{Re}=\rho_{g} v_{\text {set }} d_{p} / \mu<1\right)$ and particles larger than $10^{-9} \mathrm{~m}$. According to AVR data [4], the largest particle expected in Pebble Bed Reactors will be around 8 . $10^{-6} \mathrm{~m}$ of diameter. For these particles, the estimated value of $\operatorname{Re}$ is about $5 \cdot 10^{-6}$, which is well within the applicable range of the model.

(ii) Diffusive deposition onto surfaces is customarily modelled assuming that the turbulent flow in the gas bulk provides a uniform concentration everywhere beyond a thin boundary layer next to the surface. In the boundary layer, of thickness $\delta_{\text {dif }}$, flow is laminar and the concentration is assumed to decrease linearly to zero at the surface. Under these conditions the deposition velocity is given by $v_{\text {dif }}=$ $D / \delta_{\text {dif }}$. Using the Stokes-Einstein equation for the diffusion coefficient the velocity is expressed as [6]

$$
v_{\text {dif }}=\frac{k \cdot T_{g} \cdot C_{C}}{3 \pi \cdot \chi \cdot \mu_{g} \cdot d_{p} \delta_{\text {dif }}}
$$

The hypotheses used in the derivation of this expression are independent from the gas nature. Thus, there is no inconsistency in applying it to the HTR scenario.

(iii) The depletion due to thermophoresis (i.e., driven by temperature gradient between gas and wall surfaces) is obtained from theory using a perturbation approach with appropriated boundary conditions [7]. Talbot et al. [8] proposed a fitting formula useful for the entire range of Knudsen numbers within a $20 \%$ error margin for the available data

$$
v_{\mathrm{tph}}=\frac{2 \cdot C_{s} \cdot \mu_{g} \cdot C_{C} \cdot\left(C_{t} \cdot \mathrm{Kn}+\lambda_{g} / \lambda_{p}\right) \cdot \nabla T}{\chi \cdot \rho_{g} \cdot T_{g} \cdot\left(1+3 \cdot \mathrm{Kn} \cdot C_{m}\right) \cdot\left(1+2 \cdot C_{t} \cdot \mathrm{Kn}+2 \lambda_{g} / \lambda_{p}\right)}
$$

Parameters $C_{t}$ and $C_{m}$ depend on the gas and particle nature whereas $C_{s}$ is practically independent of the gas [9]. Based on the kinetic gas theory, Talbot et al. [8] recommended values of $C_{t}=2.18, C_{m}=1.14$, and $C_{s}=1.17$.

Since the expected temperature gradients in the confinement will be approximately $50 \mathrm{~K}$, thermophoretic deposition will not play a significant role.

2.2.2. Agglomeration Models. Both codes used in this work have the same model for the different contributions to the collision and coagulation of two particles: Brownian, Turbulent, and Gravitational.

(i) The Brownian agglomeration coefficient is given by

$$
\beta_{\text {Brow }}=\frac{2 \pi \cdot\left(D_{i}+D_{j}\right) \cdot\left(\gamma_{i} \cdot d_{i}+\gamma_{j} \cdot d_{j}\right)}{F} .
$$

This expression is derived semiempirically $[3,10]$ for the continuum regime up to the transition regime (i.e., $\mathrm{Kn}<10$ ). 
(ii) The Turbulent agglomeration coefficient was derived by Saffman and Turner [11] in terms of the energy dissipation rate, $Q_{T}$, and it consists of two contributions: the shearing and inertial part, respectively,

$$
\begin{aligned}
\beta_{T 1}= & \sqrt{\frac{\pi^{2} \cdot Q_{T}}{120 \cdot v_{g}}}\left(\gamma_{i} \cdot d_{i}+\gamma_{j} \cdot d_{j}\right)^{3}, \\
\beta_{T 2}= & \frac{0.04029 \cdot \rho_{g}^{1 / 4} \cdot Q_{T}^{3 / 4}}{\mu_{g}^{5 / 4}}\left(\gamma_{i} \cdot d_{i}+\gamma_{j} \cdot d_{j}\right)^{3} \\
& \cdot C_{\text {grav }} \cdot\left|\frac{\rho_{i} \cdot C_{C, i} \cdot d_{i}^{2}}{\chi_{i}}-\frac{\rho_{j} \cdot C_{C, j} \cdot d_{j}^{2}}{\chi_{j}}\right|,
\end{aligned}
$$

where the total coefficient is calculated as $\beta=\sqrt{\beta_{T 1}^{2}+\beta_{T 2}^{2}}$. Expressions (5) and (6) were derived on theoretical grounds and do not seem to be subject to any restriction for their application, except that particles should be smaller than the eddies responsible for their relative motion.

(iii) The Gravitational coefficient depends on the relative settling velocity of the two colliding particles by [12]

$$
\beta_{\text {grav }}=C_{\text {grav }} \cdot \frac{\pi}{4} \cdot\left(\gamma_{i} \cdot d_{i}+\gamma_{j} \cdot d_{j}\right)^{2} \cdot\left|v_{\text {set }, i}-v_{\text {set }, j}\right|,
$$

and its range of applicability is the same as for the aerosol deposition (i.e., $\operatorname{Re}<1$ ).

2.3. Intercompartmental Gas Flow Model. Generally, ASTEC and CONTAIN simulate the thermal-hydraulic behaviour of the confinement in a similar way. Nonetheless, a closer analysis of these codes reveals small but relevant differences in the model for the gas flow rate between adjacent compartments. The ASTEC code includes two mechanisms for gas mass flow [13]. The first mechanism, based on the equation of momentum, is driven by pressure gradients accordingly to

$$
\dot{G}_{\mathrm{conv}}=\frac{A_{s-t}}{L_{s-t}}\left(\Delta P_{s-t}-\zeta \frac{G|G|}{2 \bar{\rho} A_{s-t}^{2}}\right),
$$

and the second, the diffusion term, is generated by differences in concentration of each gas component and given as

$$
\dot{G}_{\mathrm{dif}, c}=\frac{D_{c} A_{s-t}}{L_{s-t}} \frac{M_{c}}{10^{3} R} \frac{d}{d t}\left(\frac{\Delta p_{c, s-t}}{\bar{T}}\right)
$$

However, in CONTAIN [2] the flow is estimated only by (8). Thus, each code estimates different fractions of incoming hot helium to be mixed with the existing gas in a compartment as well as different gas transfers to surrounding chambers. In this way, the gas flow patterns between confinement compartments are different in both codes and, consequently, the in-compartment molar fraction of gases and the net aerosol amount carried by gases. ASTEC predicts a balanced mix of gases in the confinement whereas in CONTAIN the gas mixing is not as uniform. The difference in code results will be discussed in this paper.
2.4. Gas Properties. The atmosphere in the confinement will contain a fraction of helium that, in the large break scenario, can reach significant levels for a short period of time in the discharge and adjacent compartments. Thus, not considering helium in the calculation of gas properties will lead to a deviation in the agglomeration and depletion predictions. This influence on deposition velocity can be estimated with gas properties: basically the viscosity, $\mu$, and the Cunningham factor, $C_{C}(1)$ and (2). For pure helium the viscosity is about $10 \%$ higher than for air in the range of pressures and temperatures representative of HTR accidents and $C_{C}$ will be about 20\% higher for the smallest particle (with Kn 0.2 ). Thus, the dependency of gravitational and diffusive deposition on $C_{C} / \mu$ would lead to a maximum difference of about $10 \%$ depending on the particle size. Dependency of thermophoretic deposition (3) on gas density would lead to a more significant effect ( 5 times higher for a pure helium atmosphere) in the case that this mechanism had been important.

For agglomeration models, the same approach has been taken and results for pure helium and pure air atmospheres differ to the order of $10 \%$ for Brownian and gravitational agglomeration (4) and (7). For turbulent agglomeration models, the shearing part (5) is similar for both atmospheres and the inertial part (6) is about 30\% lower in helium.

Considering that gas composition will actually be a mixture of air and helium with the fraction depending on the released helium mass flow rate (i.e., breach size), it can be concluded that the error introduced in the calculation, by not considering helium in gas atmosphere, is acceptable for these kinds of simulations.

\section{Analysis Approach: Helium Pressure Boundary Breaks}

3.1. Scenario Description. A break in the Helium Pressure Boundary (HPB) will lead to a primary circuit depressurization and the injection of helium into the Power Conversion Unit (PCU) building area. This helium injection will result in the pressurization of the specific compartment, where the break is located. The flow connection between PCU compartments will distribute the excess helium to other rooms. If the break is large enough, the Pressure Relief System (PRS) directs the helium flow towards the Depressurization Vent Shaft (DVS) system, through which the gas is released to the environment. Filter chambers at the top of the building, before the vent stack, can retain aerosols carried by the gas to limit the release of radioactive material to the environment. The DVS is connected to the PCU building through rupture panels that break if the pressure differential reaches $5 \mathrm{kPa}$.

Two different accident sequences belonging to the Licensing Basis Events (LBEs) have been simulated. Both of them consist of a breach in the HPB. The first LBE modelled is a large break (LB), $230 \mathrm{~mm}$ double ended guillotine break in the PCU piping directly coupled to the core at the bottom of the reactor. The second accident event modelled is a small break (SB), $10 \mathrm{~mm}$ diameter, located at the precooler inlet. 


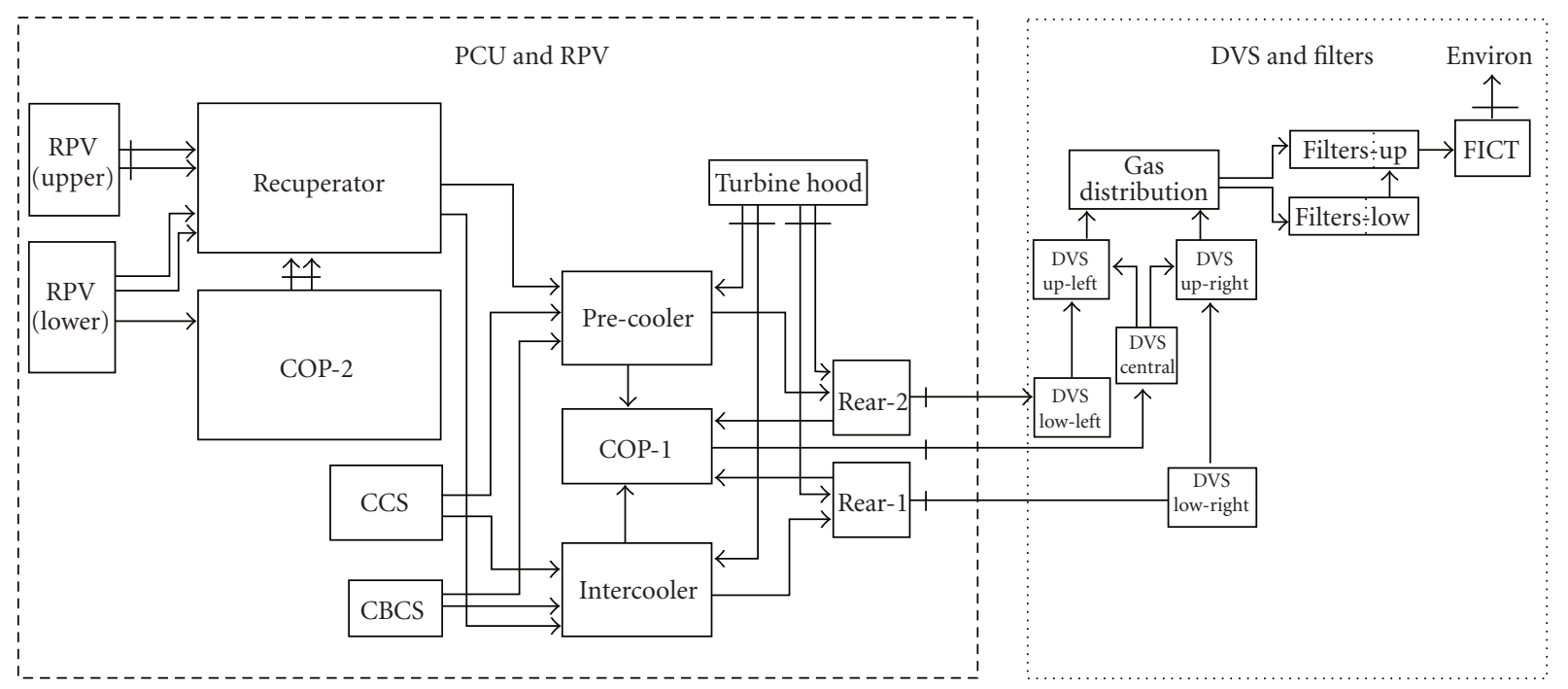

Figure 1: Diagram of the confinement building layout, including the PCU, RPV, and DVS, and the flow path connection between compartments.

The confinement building modelled in this study has a total volume of about $35000 \mathrm{~m}^{3}$ with the PCU, DVS, and filtration chambers included. The PCU is divided into 12 volumes including the Reactor Pressure Vessel compartments. The DVS comprises 8 volumes, which collectively lead to two filter compartments and these filter compartments are further split into a total of 24 chambers. This gives a total number of 42 volumes to be modelled in the ASTEC v1.3 and CONTAIN 2.0 codes. Figure 1 shows the nodalization diagram of the confinement model and the flow path between compartments.

3.2. Analytical Tools. ASTEC is an integral code devoted to the simulation of LWR severe accidents phenomena from the initiating event to the possible release of radioactive isotopes to the environment. It is being developed by IRSN (France) and GRS (Germany) helped in part by projects of the European Union research work programme. Several modules are integrated in ASTEC, of which CPA simulates the containment behaviour in accident scenarios. CONTAIN is a system code developed by the US National Regulatory Commission and has been extensively used in containment accident analysis in LWRs. Both codes use lumped parameter models to simulate the thermal-hydraulics and aerosol phenomena in multicompartment containments.

3.3. Hypotheses and Approximations. As far as is feasible, the same hypotheses and approximations adopted in the confinement modelling (i.e., compartment geometry, intercell junctions, and structure thermal properties) have been adopted in ASTEC v1.3 and CONTAIN 2.0.

Helium mass escaping the HPB through the breach is estimated with FLOWNEX [14]. Aerosols present in the primary circuit at the time of the break are carried by helium into the confinement. The helium flow rate, together with entrained aerosol mass rate, is given as a boundary condition in input decks. The dust mass entering the confinement is estimated as a fraction of the total aerosol mass accumulated within the HPB during operation. An approximation of the resuspended fraction from the circuit surfaces is calculated with the Shear Ratio Model [15] using flow conditions determined for each break size.

For the large break (LB) scenario the primary circuit depressurization is predicted to last 10 seconds. Figure 2 shows the evolution of the helium flow calculated with FLOWNEX [14]. The mass flow is very high at the beginning of the break and then decreases quickly as the pressure differential driving force between the circuit and the confinement dissipates. The helium temperature drops in a few seconds. This behaviour is due to the break location, assumed to be in the hot part of the circuit. As a consequence, the hot helium closest to the break escapes first followed by helium from further and cooler parts of the circuit (the steep decline in the temperature curve). The temperature rise after 2 seconds is due to the heat transfer from the core to the reduced helium mass available in the circuit.

During the small break (SB) accident sequence the helium, as well as the aerosol dust, is injected into the precooler compartment over a long period of time (more than 7 hours) at a constant rate (three orders of magnitudes lower than in the LB case).

With regards to aerosols, the particles are assumed to be spherical and nonhygroscopic. Additionally, it is worth mentioning that the particle-particle collision efficiency has been estimated according to Fuch's equation [12].

The aerosol size distribution is defined in the ASTEC input deck based on data from the AVR [4]. However, CONTAIN only accepts lognormal distributions; thus the data is approximated as the lognormal function that better fits the experimental distribution. The resulting distribution has a Mass Median Diameter (MMD) of $5.4 \cdot 10^{-6} \mathrm{~m}$ and a Geometric Standard Deviation (GSD) of 1.35. Figure 3 shows both aerosol size distributions used for ASTEC and CONTAIN calculations. As will be shown later, in spite of 


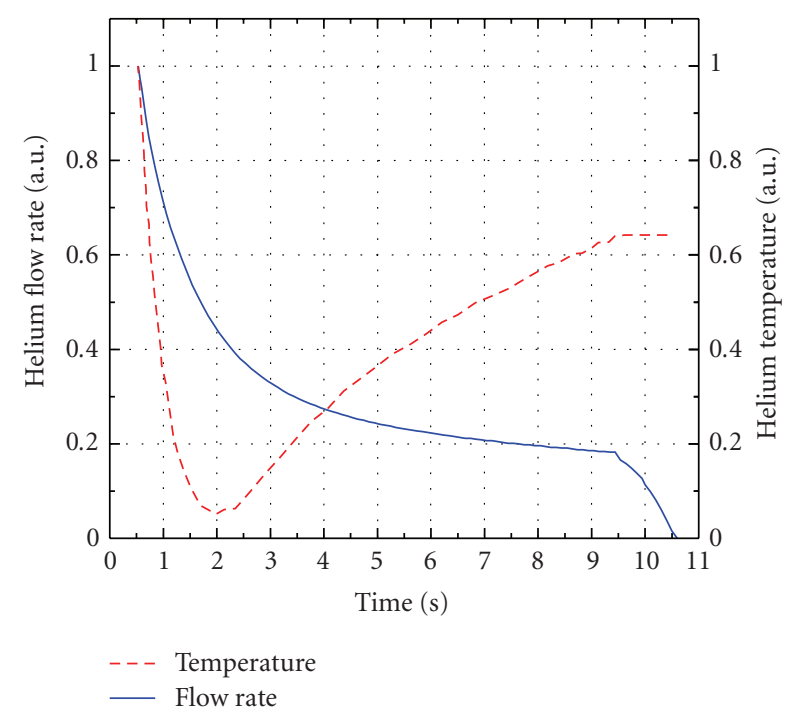

FIgURE 2: Evolution of helium flow rate and temperature escaping from primary circuit breach in the LB accident.

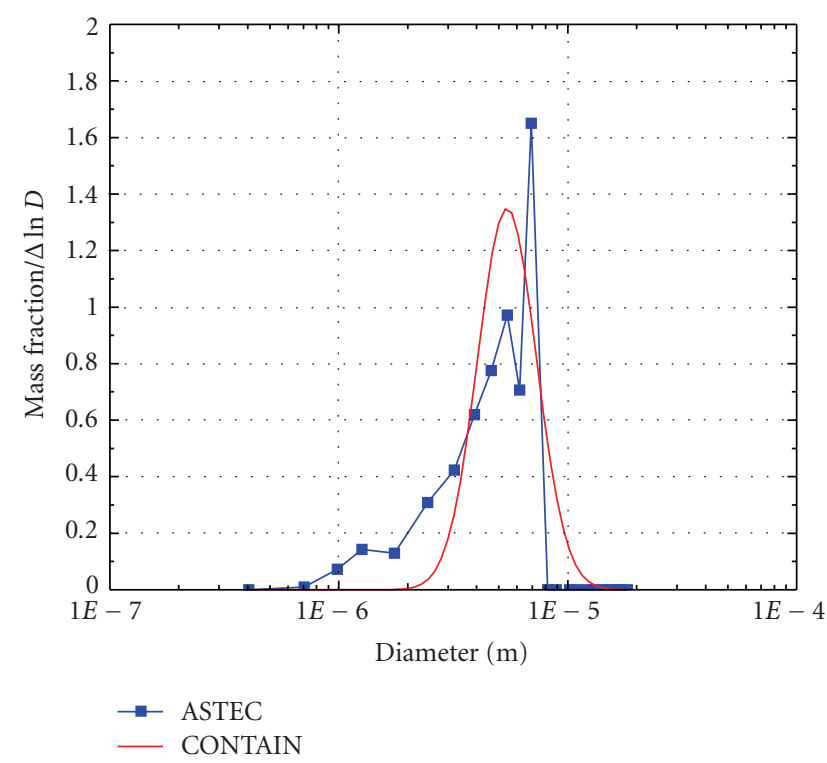

Figure 3: Aerosol size distribution defined in both code simulations.

this approximation, CONTAIN simulation results are very similar to those of ASTEC.

The total dust mass estimated to exit the HPB during the large and the small break is assumed to enter into the confinement at the same rate as the helium flow (i.e., the carrier gas). This assumption implies that the total amount of escaping dust is actually circulating in the primary circuit at the moment the accident begins.

\section{Results and Discussion}

The results below should be considered as exploratory. The approximation made above and the nonreactor specific characteristics put the emphasis of this study on its qualitative nature (i.e., trends and orders of magnitude) more than in the precise number presented below. A quantitative assessment would require a set of parametric and sensitivity calculations on all those uncertain variables discussed above.

The main thermal-hydraulic figures of merit from the point of view of the confinement safety are the gas pressure and temperature of the different compartments. Furthermore, the evolution of the accident (i.e., the confinement pressurization and helium release to the environment) is governed by the gas flow rate between adjoining compartments. Differences in the code models can drive differences in gas flow rates but also in flow patterns inside the confinement building.

Regarding the aerosol behaviour, the main safety relevant figures of merit are the aerosol mass released to the environment and the concentration remaining suspended in the confinement at the end of the accident. The rest of the aerosol mass coming into the confinement (the confinement inventory defined as "c.i.") will be depleted there, either on the filters or on compartment walls.

4.1. Large Break Scenario. In the large break sequence the primary depressurization is very fast and the injection of helium into the turbine hood compartment is quite vigorous, so two periods can be distinguished in the confinement pressurization: the first period extends from the initiation of the helium blow down to the break of the panels between turbine hood and the rest of the PCU building; the second is the subsequent homogeneous pressurization of the entire confinement. Once the HPB is depressurized, the helium blow down stops and the confinement pressure decreases as the gas is released to the environment.

4.1.1. Thermal-Hydraulics. The high helium injection during the first 0.5 seconds after the break yields a sudden pressurization of the turbine hood compartment. As a consequence of pressure differences between the turbine hood and adjacent compartments, the rupture panel connections break and helium is distributed to the other PCU compartments. As the injection flow into the turbine hood is higher than the distribution flow from the turbine hood, the pressure in the turbine hood cell increases faster than in the other volumes. This results in a sharp local peak in the turbine hood pressure. Afterwards, the inter-compartment flow is higher than the helium injection mass rate and the pressure in the PCU compartment homogenizes that is; the turbine hood depressurizes and the pressure rises in the other PCU and DVS compartments. Figure 4 plots the ratio of the actual pressure to the initial pressure for different compartments. As observed, even though the PCU-DVS and filters-environment rupture panels break soon after the onset of the accident, the pressure peaks in the confinement at about 7 seconds. After the end of the helium injection (10.5 seconds) the PCU depressurization is so fast that at about 20 seconds the confinement pressure is approximately equal to the initial pressure (Figure 4).

Both codes predict very similar evolutions of the pressure through the different periods described above. This means that both codes predict very similar flow rates during this 


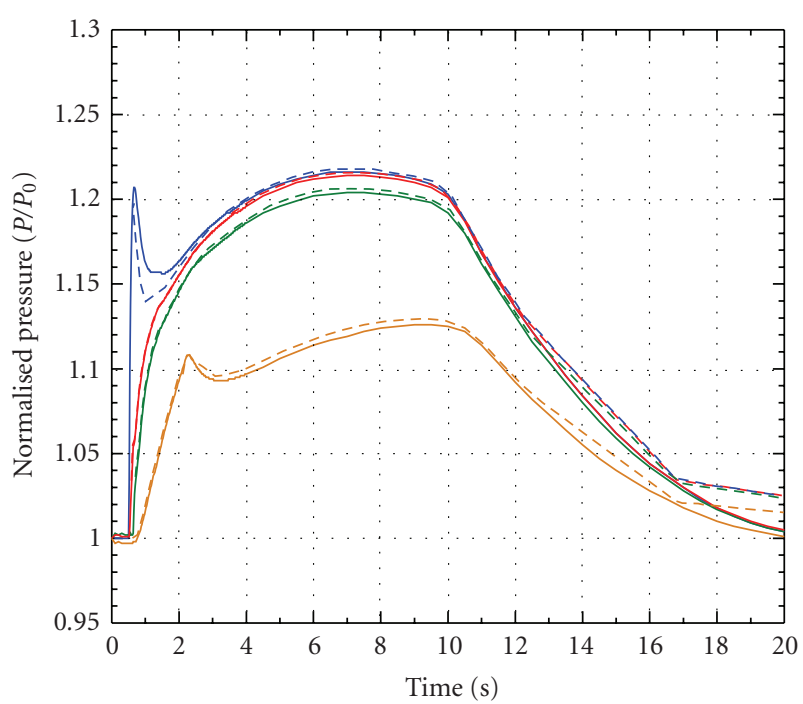

CONTAIN

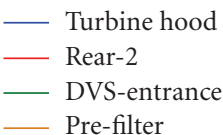

ASTEC

- - - Turbine hood

- - Rear-2

- - DVS-entrance

- - Pre-filter

Figure 4: Pressure evolution in the main confinement compartments for the LB sequence.

sequence despite their model differences. That is, the diffusion flow is negligible compared with the convection flow.

Generally speaking, temperature follows pressure evolution since hot helium is the main heating mechanism, although the thermal inertia and heat transfer to the walls and structures result in the temperature evolution being slightly slower than the pressure evolution. Figure 5 shows the temperature evolution normalized to its maximum increment. In the turbine hood the temperature quickly increases, reaching a peak at 0.8 seconds. The temperature in other compartments behaves similarly to the trend in the turbine hood but with a smoothed evolution, which is more significant at volumes far from the discharge compartment (e.g., the filters) and for the largest ones (as in the recuperator).

As shown in Figure 5, both codes predict the same temperature evolution for the entire compartment. This fact reinforces the interpretation that the effect of ignoring the diffusive term in the evaluation of the gas flow rate is negligible in this sequence.

4.1.2. Aerosols. The aerosol concentration in a given compartment is governed by the inflow mass rate (carried by the inlet helium) and by losses due to deposition on walls and to the outlet mass rate to adjoining compartments. The highest concentration is expected in the discharge compartment, the turbine hood, whose maximum concentration $(0.016 \%$ of c.i) is reached at 3.04 seconds. The compartments surrounding this chamber receive aerosols from it at a higher rate than those further away, for example, the DVS entrance or filter chambers. Thus, aerosol concentrations in the latter compartments are lower (see Figure 6). It is
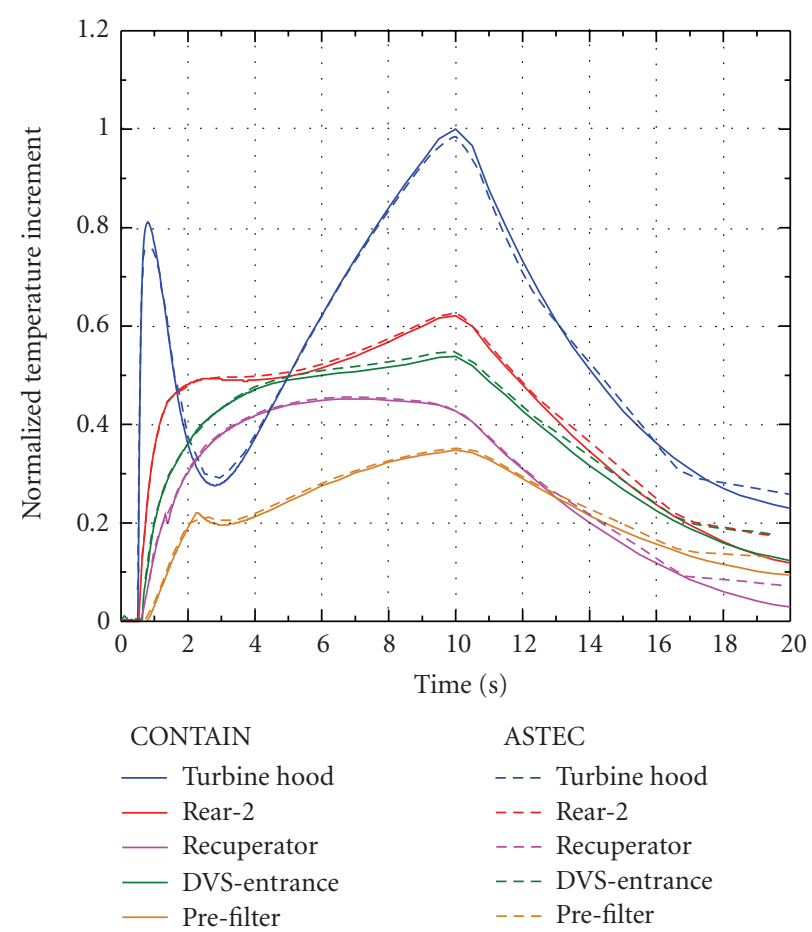

$$
\begin{aligned}
& \text { ASTEC } \\
& --- \text { Turbine hood } \\
& --- \text { Rear-2 } \\
& --- \text { Recuperator } \\
& --- \text { DVS-entrance } \\
& --- \text { Pre-filter }
\end{aligned}
$$

FIgURE 5: Temperature evolution in the main confinement compartments for the LB sequence.

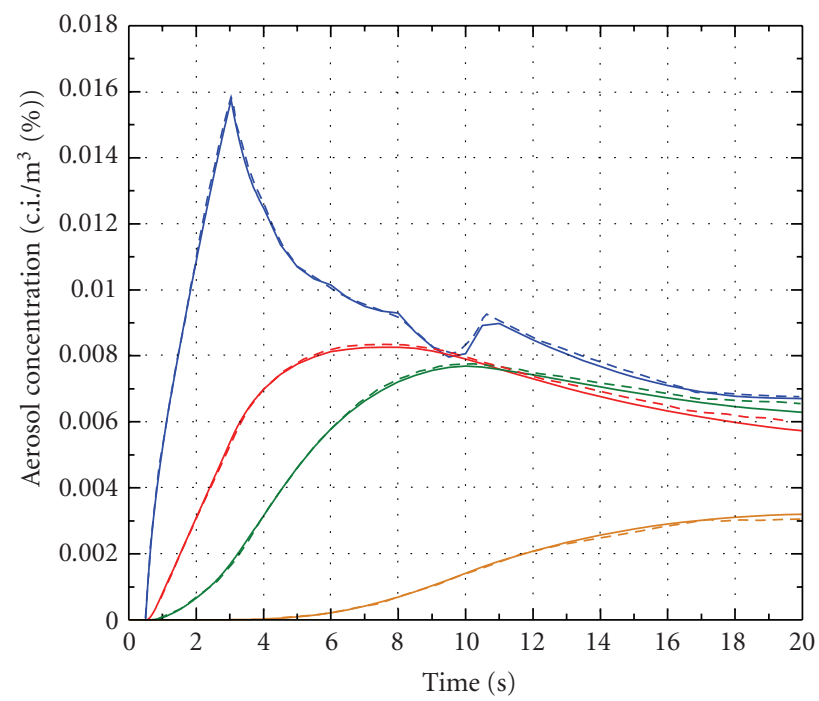

$$
\begin{array}{ll}
\text { CONTAIN } & \multicolumn{1}{c}{\text { ASTEC }} \\
\text { - Turbine hood } & --- \text { Turbine hood } \\
\text { - Rear-2 } & --- \text { Rear-2 } \\
\text { DVS-entrance } & --- \text { DVS-entrance } \\
\text { Drefilter } & -- \text { Prefilter }
\end{array}
$$

FIGURE 6: Airborne aerosol concentration evolution in the main confinement compartments for the LB sequence.

important to note that in the short term, that is, during the injection period and shortly after it, ASTEC and CONTAIN predict a very similar concentration evolution for all the compartments.

At a later time (between 50 seconds and 400 seconds), CONTAIN predicts higher concentrations in the turbine 


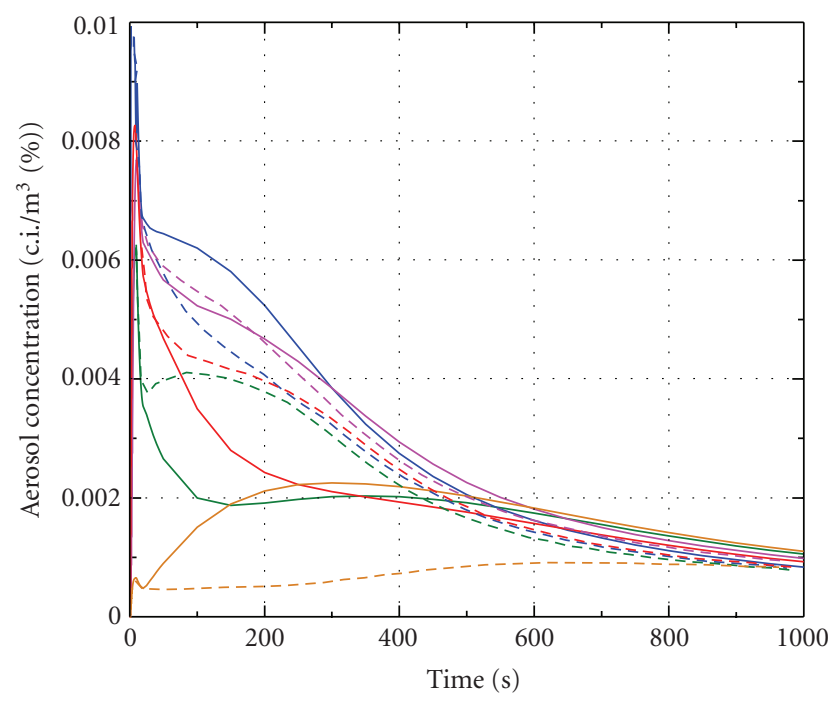

$\begin{array}{ll}\text { CONTAIN } & \text { ASTEC } \\ \text { Turbine hood } & --- \text { Turbine hood } \\ \text { Precooler } & --- \text { Precooler } \\ \text { Recuperator } & --- \text { Recuperator } \\ - \text { Rear-2 } & --- \text { Rear-2 } \\ \text { DVS-entrance } & --- \text { DVS-entrance }\end{array}$

FIgURE 7: Airborne aerosol concentration evolution in the main confinement compartments for the LB sequence (long term simulation).

hood (around 30\%) and lower concentrations in the rear compartments and precooler/intercooler volumes (30\% and $50 \%$ lower, resp.), compared with ASTEC estimates. Nevertheless, at about 500 seconds the two simulations converge as is shown in Figure 7. This behaviour can be explained by the differences in the inter-compartment flow model rather than by differences in aerosol modelling. During the first phase of the accident the gas flow is mainly driven by pressure gradients but at about 20 seconds the pressure in the confinement reaches a steady value and the flow between compartments is mainly due to diffusion. Given the absence of the diffusive term in the CONTAIN momentum equations, the intercell flow predicted from then on by ASTEC is different to that of CONTAIN and, as a consequence, the net aerosol mass entering compartments is also different. Furthermore, the particle size distribution is influenced too since agglomeration is proportional to the particle concentration squared and the dominant depletion mechanisms, such as sedimentation, affect large particles preferentially.

In spite of these code-to-code differences in the inconfinement aerosol evolution, they hardly affect the aerosol mass balance at the end of the simulation (Table 1). Both codes predict that most of the injected mass (more than 60\%) is deposited on the confinement walls and that most of this mass (above 95\%) is depleted on the floors by gravitational settling. The second contribution to the aerosol mass balance is the aerosol remaining suspended in the confinement building at the end of the simulation (between 20-30\%). The total mass released to the environment represents only about

TABLE 1: Aerosol mass balance for the LB sequence.

\begin{tabular}{lcc}
\hline & $\begin{array}{c}\text { ASTEC } \\
\text { (\% of c.i.) }\end{array}$ & $\begin{array}{c}\text { CONTAIN } \\
\text { (\% of c.i.) }\end{array}$ \\
\hline Airborne mass in the confinement & 20.1 & 27.3 \\
Released mass to the environment & 0.6 & 1.6 \\
Deposited mass on walls & 61.4 & 64.8 \\
Deposited mass in filters & 17.9 & 14.4 \\
\hline
\end{tabular}

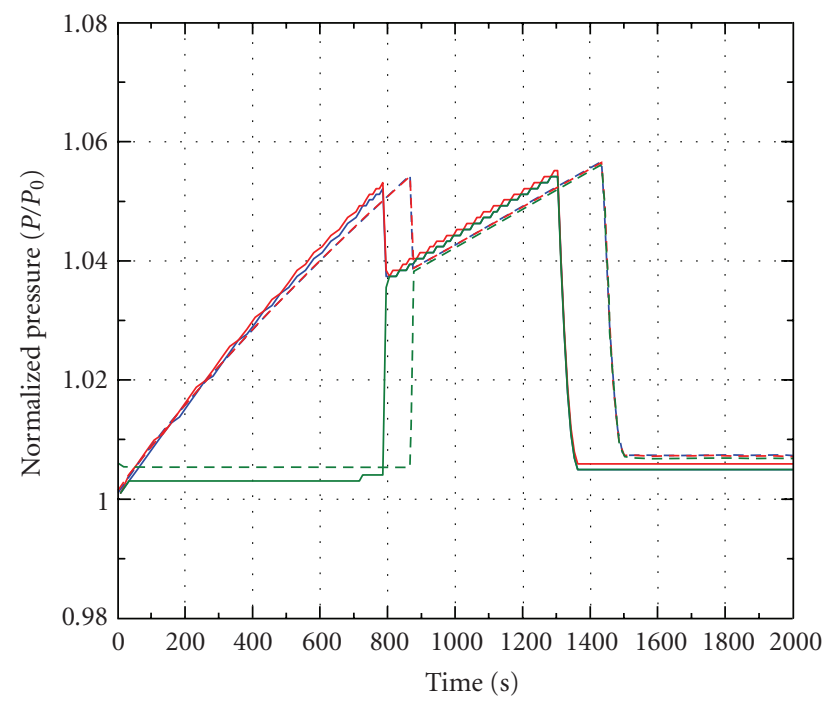

CONTAIN

$$
\begin{array}{ll}
\text { — Precooler } & -- \text { Precooler } \\
\text { - Rear-2 } & --- \text { Rear-2 } \\
\text { DVS-entrance } & --- \text { DVS-entrance }
\end{array}
$$

ASTEC:

Figure 8: Pressure evolution in the main confinement compartments for the SB sequence.

$1 \%$ of the confinement inventory $(0.6 \%$ for ASTEC and 1.6 for CONTAIN results).

Although the aerosol models in both codes originate from the MAEROS code [5], there are some differences in the capabilities of each code. The most significant is that CONTAIN does not have a model for filter retention. This will cause the main difficulty in estimating the filtered and released mass in an HTR confinement analysis; however, an ad hoc calculation can be done after code simulation. Using CONTAIN output data of the prefilter aerosol concentration, flow through the filters and based on the filter efficiency, the filtered mass is calculated via the following expression:

$$
M_{\mathrm{fil}}=\varepsilon_{\mathrm{fil}} \cdot \sum_{\text {chamber }} \sum_{t_{i}=t_{0}}^{t_{\text {end }}} C_{\text {aer }, k}\left(t_{i}\right) \cdot G_{\text {fil }, k}\left(t_{i}\right) \Delta t_{i} .
$$

This method for calculating the filter retention introduces an error in the mass balance of about $8.1 \%$.

Given the high gas flow rates attained in this scenario and the direction and cross-section changes in gas flows, particle impaction could become a significant depletion mechanism. As ASTEC and CONTAIN do not include models for this process, it should be expected that the above estimates are conservative. 


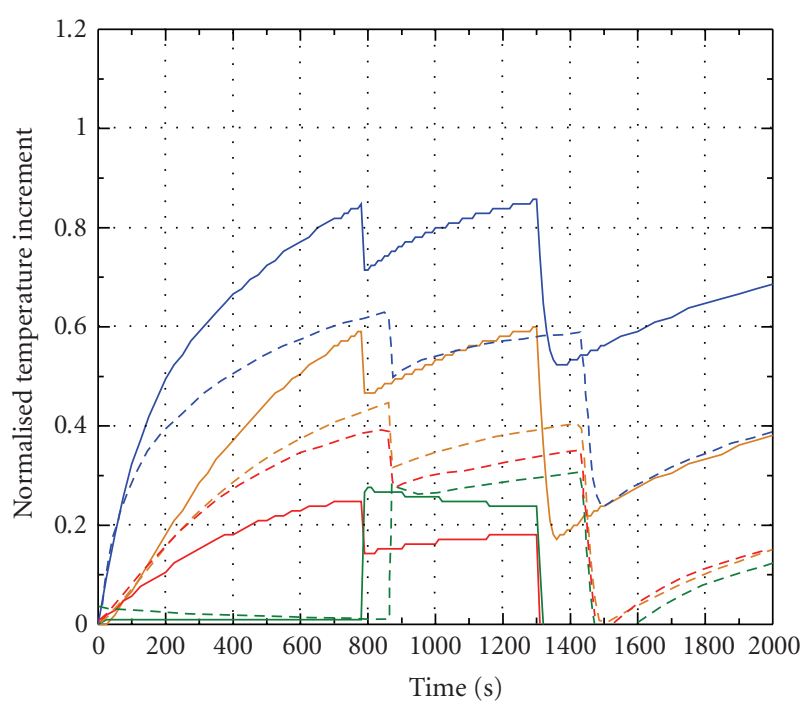

CONTAIN

ASTEC

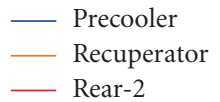

— DVS-entrance

Figure 9: Temperature evolution in the main confinement compartments for the SB sequence (short term simulation).

4.2. Small Break Scenario. The general behaviour of the confinement is very different than that in the LB accident. Slow helium blowdown into the precooler compartment yields a smooth pressure increase so that effects of helium diffusion are nonnegligible compared with the convection driven force for gas flow.

4.2.1. Thermal-Hydraulics. The helium flow entering the precooler compartment is distributed to other PCU compartments and the pressure in the PCU building increases homogenously (Figure 8). Later on, at about 800 seconds, the rupture panels between the PCU compartment and the DVS break, the gas passes through the DVS to the filters chambers, breaks the rupture panels after the filters, and passes to the environment at 1400 seconds.

Slight differences are observed in the simulation of the ASTEC and CONTAIN codes. ASTEC predicts a slightly delayed rupture of panels between the PCU and DVS and between the DVS stack and the environment (800 and 1430 seconds); whereas CONTAIN predictions are 780 and 1300 seconds, respectively (Figure 8).

As in the previous case, the intercell flow model is responsible for most of the discrepancies in the codes estimates. As a consequence of the exchange enhancement driven by the diffusive term, ASTEC predicts a more uniform gas temperature in different chambers since hot helium is capable of reaching farther from the break point. This difference together with that in mole number in each cell is the basis of the pressure discrepancies between both codes.

Figure 9 shows that in the short term the temperature evolution follows the trend of the pressure (i.e., net helium injection into the compartment). In the long term, after the
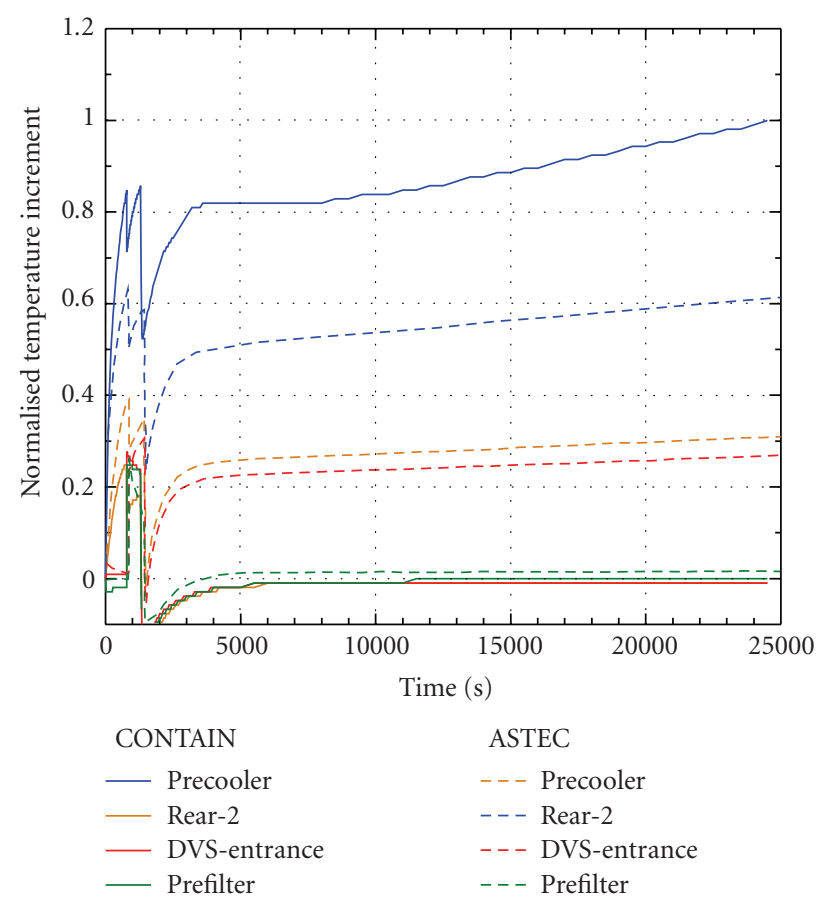

Figure 10: Temperature evolution in the main confinement compartments for the SB sequence (long term simulation).

environment rupture panels break, the temperature increases slowly due to the long helium injection and maximum temperature is reached in the precooler compartment at the end of the simulation (Figure 10).

4.2.2. Aerosols. The effect of differences in the flow models is more evident in the simulation of the aerosol mass concentration in specific compartments. As mentioned previously, one of the main variables influencing the aerosol concentration is the balance of the inlet/outlet mass flow rate carried by the gas. Figure 11 plots the aerosol concentration predictions of some of the main compartments given by ASTEC and CONTAIN. Since ASTEC predicts higher gas flows and more homogeneous helium distribution in the confinement, the aerosol mass is also distributed more homogeneously in ASTEC calculation than in CONTAIN.

In spite of these large differences in the aerosol concentration the general behaviour of aerosols is similarly predicted by both codes. The total airborne and deposited mass in the entire PCU and DVS buildings is fairly similar in both code simulations as is shown in Figure 12, with the highest difference in the airborne mass occurring in the PCU compartments.

Concerning the aerosol mass balance, both codes predict that most of the injected dust mass (about 60\%) is deposited on the confinement floor (Table 2). For ASTEC, the second contribution to the mass balance is the filter retention and thereafter the airborne mass. However, in CONTAIN more mass remains suspended in the confinement than retained in filters. The aerosol release to the environment is about $1 \%$ (0.7\% for ASTEC simulation whereas CONTAIN estimates $1.2 \%$ of c.i.). The total aerosol mass differs in both codes, as 

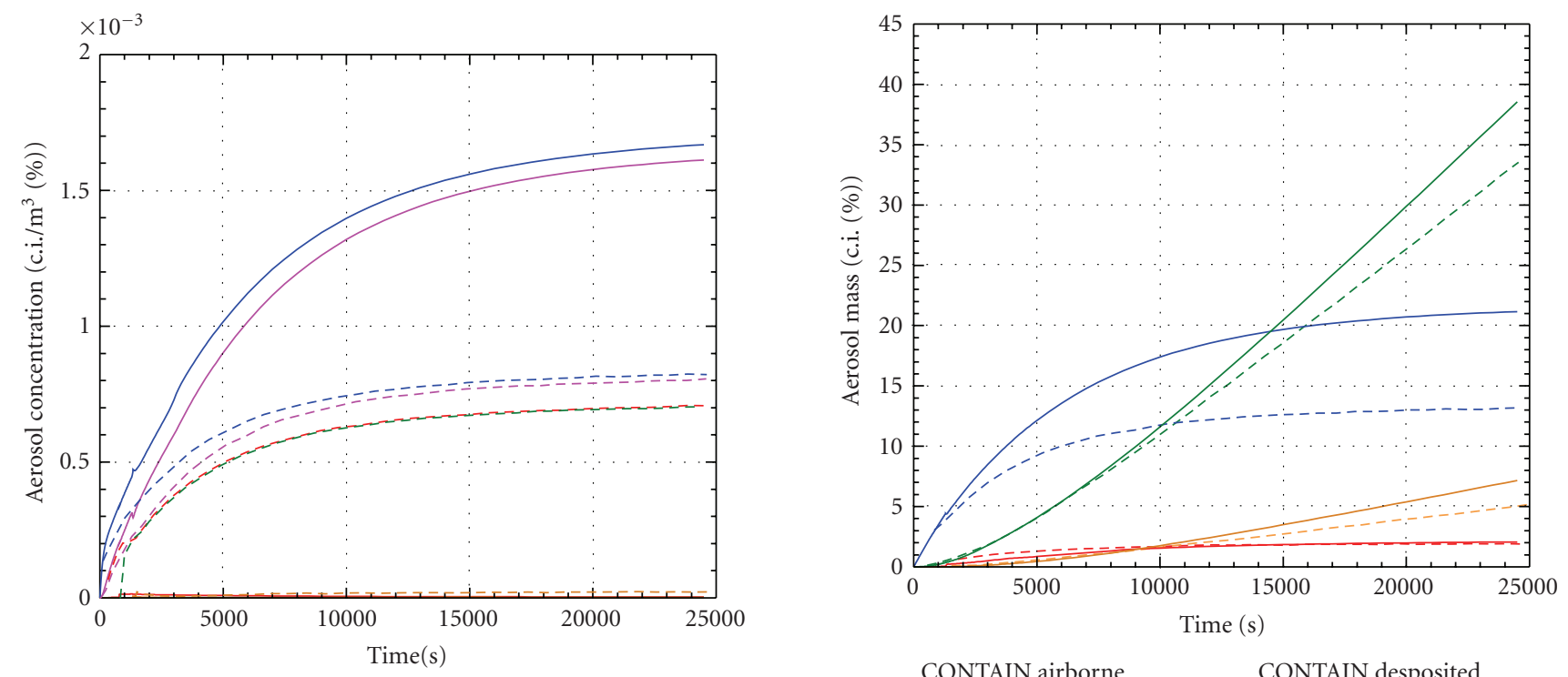

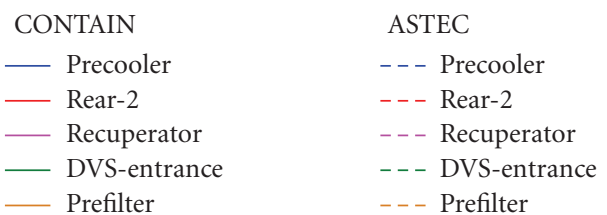

Figure 11: Aerosol concentration evolution in the main confinement compartments for the SB sequence.

TABLE 2: Aerosol mass balance for the SB sequence.

\begin{tabular}{lcc}
\hline & $\begin{array}{c}\text { ASTEC } \\
\text { (\% of c.i.) }\end{array}$ & $\begin{array}{c}\text { CONTAIN } \\
\text { (\% of c.i.) }\end{array}$ \\
\hline Airborne mass in the confinement & 16.3 & 24.7 \\
Released mass to the environment & 0.7 & 1.2 \\
Deposited mass on walls & 61.0 & 56.8 \\
Deposited mass in filters & 22.0 & 10.6 \\
\hline
\end{tabular}

in the case of the LB, because of the ad doc calculation of filter retention. In this calculation the difference is about $6.7 \%$ of the confinement inventory (Table 2).

For the SB accident, the aerosol mass entering the confinement is 2000 times lower than in the LB scenario and the injection time extends over more than 7 hours. However, comparison of Tables 1 and 2 shows that the aerosol mass balance, in terms of relative mass, is qualitatively similar in both scenarios.

\section{Conclusions}

Safety is a key aspect of nuclear systems development. Any innovative system, particularly HTR ones, requires a thorough safety study to be carried out, which includes accident analyses to demonstrate compliance with regulatory dose and risk limits for both the public and the worker during accident conditions. Confinement building performance is, therefore, a key element of the system. This paper has jointly explored two important issues: available simulation

$$
\begin{array}{ll}
\text { CONTAIN airborne } & \text { CONTAIN desposited } \\
- \text { PCU + RPV } & - \text { PCU + RPV } \\
- \text { DVS } & \text { DVS } \\
\text { ASTEC airborne } & \text { ASTEC desposited } \\
--- \text { PCU + RPV } & --- \text { PCU + RPV } \\
--- \text { DVS } & --- \text { DVS }
\end{array}
$$

FIGURE 12: Airborne and deposited mass evolution in the confinement for the SB sequence.

capabilities at hand and performance of postulated HTR confinement under prototypical accident conditions.

ASTEC and CONTAIN, although mainly developed in LWR field, have been shown to be largely applicable to HTR confinement scenarios. An estimate of the Knudsen number in expected conditions under HPB breaks accidents indicates that these scenarios lie in the continuum or slip flow regimes, well inside the range of validity of the models encapsulated within both codes.

Additionally, their simulation capabilities have been illustrated by comparing their response under two accident scenarios with breaks in the HPB: a large and a small break. In both sequences, the same hypotheses and approximations have been used for both code simulations. The main conclusions for this comparison from a thermal-hydraulic point of view are the following.

(i) ASTEC v1.3 and CONTAIN 2.0 predict very similar confinement performance in both magnitude and time.

(ii) Differences have been found in the intercompartment gas flow modelling. These differences affect the gas composition in specific compartments but they do not result in any substantial discrepancy in the overall variables characterizing the scenario. The effect of these flow differences is more significant in the small break accident, where the injected helium and intercell flows are smaller and the flow is driven mainly by diffusion.

The main observations regarding aerosol behaviour are as follows. 
(i) Most of the aerosol inventory coming into the confinement is depleted on the walls and only about $1 \%$ of the aerosol mass is released to the environment.

(ii) ASTEC v1.3 and CONTAIN 2.0 predict similar evolution of aerosols in the confinement, especially in the large break accident, even though the injected size distributions used in both codes are not entirely the same.

(iii) Minor differences in the aerosol concentration evolution in specific compartments have been found due to the differences in inter-compartment gas flow code estimates.

With regard to the availability of models, ASTEC has a specific model for aerosol retention in filters whereas CONTAIN does not have this feature available. This requires the user to set up an independent way of estimating the filtration effect in the case of CONTAIN. On the other hand, in the scenarios analysed, CONTAIN has demonstrated to be more robust under a large step injection of gas and aerosol.

Finally, even though the absence of a model for depletion by impaction in both codes leads to conservative results, in further studies it is recommended that the quantitative effect of this mechanism to be assessed.

\section{Nomenclature}

A: $\quad$ Flow path section

D: Diffusivity

$C$ : Concentration of aerosols

$C_{C}$ : Cunningham correction factor

$C_{\text {grav }}$ : Gravitational collision efficiency

$C_{m}$ : Constant

$C_{s}$ : Constant

$C_{t}$ : Constant

d: $\quad$ Particle diameter

F: $\quad$ Correction factor

$G$ : $\quad$ Flow rate

g: $\quad$ Gravitation constant

$k$ : $\quad$ Boltzmann constant

Kn: Knudsen number

L: $\quad$ Flow path length

M: Aerosol mass

$P$ : $\quad$ Pressure

$Q_{T}$ : Dissipation rate of the turbulent energy

$R$ : Universal gass constant

T: $\quad$ Temperature

$t$ : Time step

$v$ : Velocity

\section{Greek}

$\beta$ : Agglomeration coefficient

$\gamma$ : Agglomeration shape factor

$\delta$ : Boundary layer thickness

$\varepsilon$ : Retention efficiency

$\lambda$ : Average free path length

$\lambda$ : Heat conductivity

$\mu$ : Dynamic viscosity $v$ : Kinematics viscosity

$\rho$ : Density

$\chi$ : Dynamic shape factor

$\zeta$ : Flow resistance

\section{Subscripts and Superscripts}

\author{
aer: Aerosol \\ Brow: Brownian \\ c: Gas componenet \\ con: Convective \\ dif: Diffusion \\ dph: Diffusiophoresis \\ fil: $\quad$ Filter \\ g: $\quad$ Gas atmosphere \\ grav: Gravitational \\ $i$ : $\quad$ Particle $i$ \\ $j$ : $\quad$ Particle $j$ \\ $k$ : $\quad$ Filter chamber index \\ $p: \quad$ Particle \\ $s: \quad$ Source compartment \\ set: Settling \\ $t$ : Target compartment \\ tph: Thermophoresis
}

\section{List of Acronyms}

CFP: Coated fuel particle

DVS: Depressurization vent shaft

HPB: Helium pressure boundary

HTR: High temperature reactor

LB: Large break

LWR: Light water reactor

MMD: Mass median diameter

PCU: Power conversion unit

PRS: Pressure relief system

RPV: Reactor pressure vessel

SB: $\quad$ Small break

\section{Acknowledgment}

This work has been developed under the PBMR-CIEMAT agreement for confinement analysis.

\section{References}

[1] J. P. Van Dorsselaere and B. Schwinges, "Overview of the integral code ASTEC V1.3," IRSN Technical Note DPAM/ASTEC 2006-362, 2006, GRS Technical Note GRS ASTEC 06/02.

[2] K. K. Murata, "Code manual for CONTAIN 2.0: a computer code for nuclear reactor containment analysis," NUREG/CR6533, SAND97/1735, 1997.

[3] M. Sitarski and J. H. Seinfeld, "Brownian coagulation in the transition regime," Journal of Colloid And Interface Science, vol. 61, no. 2, pp. 261-271, 1977.

[4] U. Wawrzik, P. Biedermann, and H. F. Oetjen, "Staub im AvrReaktor; Verhalten Bei Transienten Strömungsbedingungen," in Proceedings of the Jahrestagung Kerntechnik, Karlsruhe, Germany, 1987. 
[5] F. Gelbard, "MAEROS users manual," NUREG/CR-1931, SAND80-0822, 1982.

[6] W. C. Hinds, Aerosol Technology, John Wiley \& Sons, New York, NY, USA, 2nd edition, 1999.

[7] J. R. Brock, "On the theory of thermal forces acting on aerosol particles," Journal of Colloid Science, vol. 17, no. 8, pp. 768-780, 1962.

[8] L. Talbot, R. K. Cheng, R. W. Schefer, and D. R. Willis, "Thermophoresis of particles in a heated boundary layer," Journal of Fluid Mechanics, vol. 101, part 4, pp. 737-758, 1980.

[9] B. K. Annis, "Thermal creep in gases," The Journal of Chemical Physics, vol. 57, no. 7, pp. 2898-2905, 1972.

[10] N. A. Fuchs, The Mechanics of Aerosols, Pergamon Press Book, New York, NY, USA, 1964.

[11] P. G. Saffman and J. S. Turner, "On the collision of drops in turbulent clouds," Journal of Fluid Mechanics, vol. 1, pp. 1630, 1956.

[12] G. Weber, "ASTEC V0 Description of aerosol models in the containment part of ASTEC (CPA)," ASTEC-V0/DOC/99-20, 1999.

[13] W. Klein-Hessling and B. Schwinges, "ASTEC V0. CPA module program reference manual," ASTEC-V0/DOC/01-34, 1998.

[14] A. Pitso, "MPS pipe break analysis calculation report," private communication, 2007.

[15] K. Sawa, T. Nishimoto, and Y. Miyamoto, "Experimental study of dust behaviour during depressurization," Journal of Nuclear Science and Technology, vol. 29, no. 10, pp. 1018-1025, 1992. 

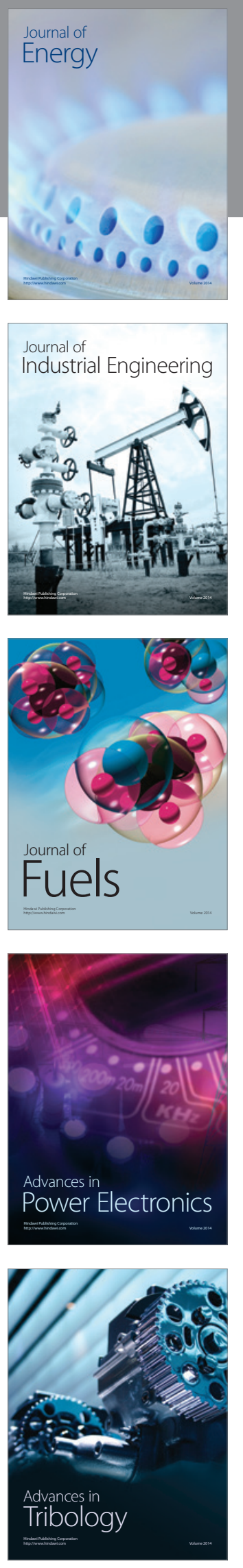
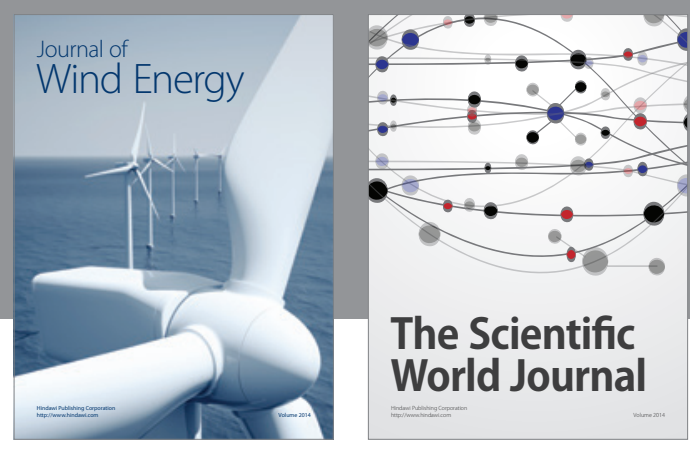

The Scientific World Journal

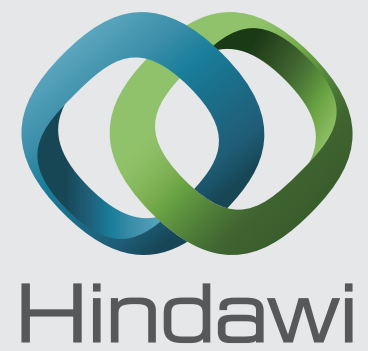

Submit your manuscripts at http://www.hindawi.com
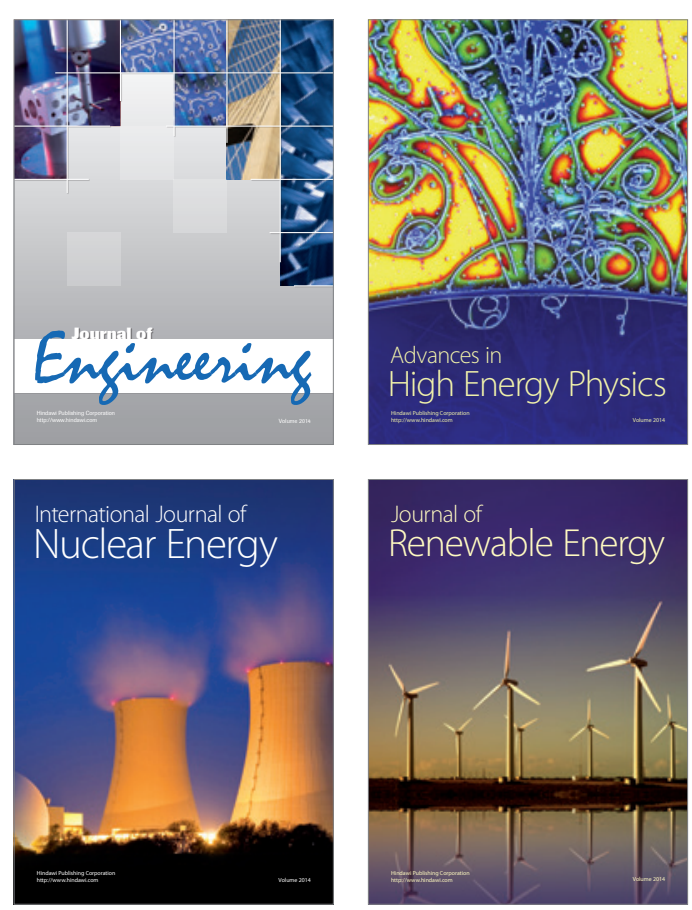

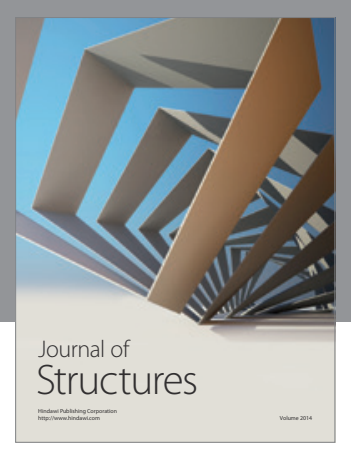

Rotating
Mechinery
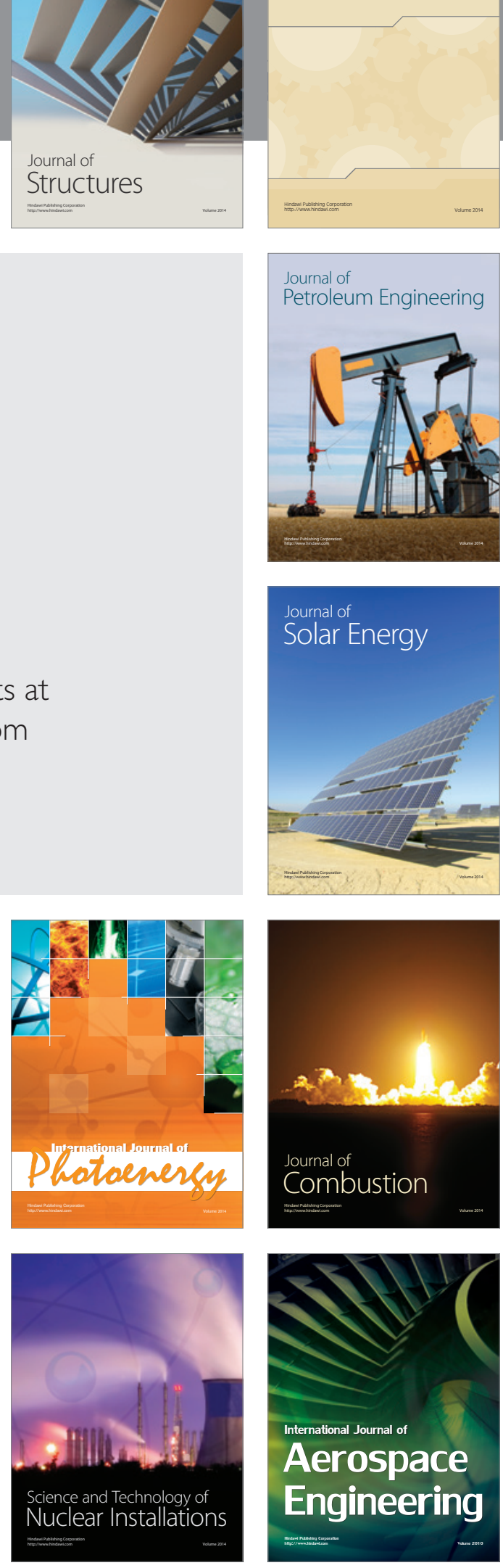